\title{
Monte Carlo Simulation of the Echo Signals from Low-Flying Targets for Airborne Radar
}

\author{
Mingyuan Man, ${ }^{1}$ Zhenya Lei, ${ }^{1}$ Yongjun Xie, ${ }^{2}$ Botao Chen, ${ }^{3}$ and Qing Wang ${ }^{1}$ \\ ${ }^{1}$ National Laboratory of Antennas and Microwave Technology, Xidian University, Xian 710071, China \\ ${ }^{2}$ School of Electronic Engineering, Beihang University, Beijing 100191, China \\ ${ }^{3}$ Science and Technology on Space Physics Laboratory, Beijing 100076, China
}

Correspondence should be addressed to Mingyuan Man; man-ming-yuan@163.com

Received 15 June 2014; Accepted 20 October 2014; Published 17 November 2014

Academic Editor: Felipe Cátedra

Copyright (C) 2014 Mingyuan Man et al. This is an open access article distributed under the Creative Commons Attribution License, which permits unrestricted use, distribution, and reproduction in any medium, provided the original work is properly cited.

\begin{abstract}
A demonstrated hybrid method based on the combination of half-space physical optics method (PO), graphical-electromagnetic computing (GRECO), and Monte Carlo method on echo signals from low-flying targets based on actual environment for airborne radar is presented in this paper. The half-space physical optics method, combined with the graphical-electromagnetic computing (GRECO) method to eliminate the shadow regions quickly and rebuild the target automatically, is employed to calculate the radar cross section (RCS) of the conductive targets in half space fast and accurately. The direct echo is computed based on the radar equation. The reflected paths from sea or ground surface cause multipath effects. In order to accurately obtain the echo signals, the phase factors are modified for fluctuations in multipath, and the statistical average value of the echo signals is obtained using the Monte Carlo method. A typical simulation is performed, and the numerical results show the accuracy of the proposed method.
\end{abstract}

\section{Introduction}

Due to Earth curvature, terrain masking, and clutter interference, low altitude targets are difficult to detect by the ground radar [1-3]. Hence, the flying targets including military aircrafts and crushing missiles can make low or ultralow altitude flying to avoid the radar detection. However, airborne early warning (AEW) radar plays a primary role in the detection of low-flying aircraft, which are out of the coverage of ground radars $[4,5]$. The AEW system also plays an important role in other aspects, such as the coordination of search and rescue and airborne rendezvous control. Owing to vast cost in an AEW system experiment and other difficulties, the simulation of the AEW system based on computers is an effective way to evaluate its performance [6-10]. An accurate estimate of the detection probability and other performances in look-down mode for airborne radar can be obtained by a simulation code that reproduces the output of the signal processor in every practical operating condition. To improve the detection performance, it is necessary to accurately obtain the echo signals from low-flying targets for airborne radar.
The problem has been studied by many other authors $[1,2,11,12]$. The work finished in $[1,2]$ has dealt with AEW radar system in detail and briefly introduced the theory of AEW radar system on evaluating the electromagnetic characteristics of the interactions between the targets and the environment. The tropospheric propagation channel background is exactly described for low-altitude surveillance, and wideband frequency is utilized to mitigate and exploit specular multipath channel characteristics in [11]. The experimental data for a variety of terrain types is presented in [13], which indicates that a statistical analysis is required for the signal strength in practice. However, the experiments are carried out just for one-way propagation. The probability density function (PDF) of target's echo in the presence of multipath effects is discussed in [12], but only two ways of echoes are considered with the flat-earth geometry. When the radar's altitude increases, some important assumptions will not be satisfied.

The statistical simulation of echo signals from low altitude targets based on actual environment for airborne radar is presented in this paper. The low-flying target RCS is calculated 
by using the quasistationary approximation. The half-space physical optics integral equation is derived by introducing the half-space Green's function into PO. Combined with the graphical-electromagnetic computing method, the RCS of conductive targets can be calculated in half space exactly and efficiently. Along with the description of the dynamic situations, the direct echo is computed based on the radar equation. There are reflected paths from sea or ground surface giving rise to multipath effects. The phase factors are modified using the Monte Carlo method in order to eliminate the fluctuations caused by multipath, and the statistical characteristics of echoes are obtained at last.

The organization of this paper is shown as follows. In the first part of Section 2, the relative incident direction is derived by coordinate transformation, and the RCS of the low-flying target is calculated by the half-space physic-optics method, combined with GRECO [14]. The second part of Section 2 describes multipath propagation modeling and introduces the Monte Carlo method to modify the phase factors of different paths for the statistical characteristics of echo signals. In Section 3, some simulations are given, as well as the results and comparisons among the simulations. Conclusion is drawn in the final section.

\section{Theory}

2.1. Dynamic RCS of Low-Fly Targets. The echo signal power is dependent on the target RCS. As the low-altitude target is flying closely to the ground or sea surface, its RCS is quite different from the one in the free space. The incident wave direction of propagation is determined by the radar-target engagement geometry and described by the azimuth angle $\varphi_{r}$ and the elevation angle $\theta_{r}$ with respect to the target bodyfixed axes. In order to calculate the target RCS of the target illuminated by the radar, $\varphi_{r}$ and $\theta_{r}$ should be achieved by coordinate transformation [15] with the given longitudes, latitudes, heights, and attitudes of the radar and the target (Table 1). When the incident wave direction of propagation is obtained, the low-flying target RCS can be exactly calculated using half-space physic-optics method $[16,17]$.

2.1.1. Half-Space PO for Radar Targets. Considering an arbitrarily shaped object illuminated by a plane wave in the half space, surface $S$ is assumed to represent a closed surface of target. The far zone scattering fields of the conductive target in half space can be expressed as

$$
E_{s}\left(r^{\prime}\right)=-j \omega A\left(r^{\prime}\right)-\nabla \Phi\left(r^{\prime}\right), \quad r^{\prime} \in S
$$

where $A$ and $\Phi$ represent the vector and scalar potentials due to the surface current $J\left(r^{\prime}\right)$, respectively.

The half-space Green's function can be expressed by the vector or scalar potentials, and the vector potential is not uniquely specified among literatures. It is expressed in this paper using the form mentioned in [18] as follows:

$$
\bar{G}_{A}=(\widehat{x} \hat{x}+\widehat{y} \hat{y}) G_{A}^{x x}+\widehat{z} \widehat{x} G_{A}^{z x}+\widehat{z} \hat{y} G_{A}^{z y}+\widehat{z} \widehat{z} G_{A}^{z z},
$$

in which

$$
G_{A}^{y y}=G_{A}^{x x}, \quad G_{y}^{q e}=G_{x}^{q e},
$$

where $G_{A}^{x x}, G_{A}^{z x}, G_{A}^{z y}$, and $G_{A}^{z z}$ denote the spatial domain half-space Green's function for the vector potentials and $G_{x}^{q e}$, $G_{y}^{q e}$, and $G_{z}^{q e}$ denote the spatial domain half-space Green's function for the scalar potentials $[19,20]$. According to the position of targets, the terrain below targets can be derived from digital feature analysis data (DFAD). Then the ground characteristic is introduced into half-space Green's function. The fluctuant ground is equivalent to the medium surface in lower half-space.

As stated before, the half-space Green's function has been introduced into PO to compute the electrically large targets in the half space. Combined with the graphical electromagnetic computing method (GRECO) [14], the geometry information of each illuminated plane is obtained by reading the colors and depths of each pixel, and the shadow regions are eliminated by displaying lists technology of OpenGL to rebuild the target. Hidden surfaces of the image have been previously removed by the hardware graphics accelerator. Then we make use of the resolution to disperse the curve face into pixels that satisfy the requirement of the electromagnetic calculation; meanwhile, the scene is rendered using the Phong local illumination model [21]. For three light sources of purely green, red, and blue colors, respectively, located over each one of the three coordinate axises, the three color components for this pixel are equal to the $\left(n_{x}, n_{y}, n_{z}\right)$ components of the unit normal to surface. Meanwhile, the depths of each pixel are obtained in the same way.

The far zone scattering fields of the conductive target in half space is expressed as

$$
\begin{aligned}
& E_{s}(r) \\
& =-j \omega \iiint_{v} \bar{G}_{A} \cdot J\left(r^{\prime}\right) d r^{\prime}+\frac{k \cdot \widehat{s}}{\omega} \\
& \quad \times \iiint_{v}\left[G_{x}^{q e} \frac{\partial}{\partial x} J\left(r^{\prime}\right)+G_{y}^{q e} \frac{\partial}{\partial y} J\left(r^{\prime}\right)+G_{z}^{q e} \frac{\partial}{\partial z} J\left(r^{\prime}\right)\right] d r^{\prime} .
\end{aligned}
$$

Equation (4) is then calculated for every illuminated facet, and the complex RCS due to scattering from every illuminated facet is calculated as [22]

$$
\sqrt{\sigma}=\lim _{R \rightarrow \infty} 2 \sqrt{\pi} R \frac{E_{s} \cdot \widehat{e}_{r}}{E_{o}} \exp (j k R),
$$

where $\exp (j k R)$ is the phase term which is introduced into the equation in order to account for the facet location with respect to the global coordinate system, and $R$ is the position vector for the facet's reference vertex with respect to the global coordinate system.

By using half-space PO to evaluate the scattering from the target in half-space, note that the use of GRECO to remove shadowed surfaces is valid for the direct field incidence direction not for ground reflections. This is valid only if the target is low flying, because the incident and ground-reflection directions are almost parallel. In this case, 
TABLE 1: Some primary parameters.

\begin{tabular}{lccc}
\hline Parameter & Value & Parameter & Value \\
\hline Radar's longitude & $123^{\circ}$ & Radar's latitude & $31.6^{\circ}$ \\
Radar's height & $7500 \mathrm{~m}$ & Target's longitude & $124.7^{\circ}$ \\
Target's latitude & $31.9^{\circ}$ & Target's height & $20 \mathrm{~m}$ \\
Radar's emission power & $1000 \mathrm{~kW}$ & Frequency & $0.4 \mathrm{GHz}$ \\
Emission signal modality & Linear frequency modulation & $13 \mu \mathrm{s}$ \\
Pulse compression ratio & $13 \mu \mathrm{s} / 220 \mathrm{~ns}$ & Emission signal pulse width & $400 \mathrm{~m} / \mathrm{s}$ \\
Antenna maximal gain & $32 \mathrm{~dB}$ & Aircraft's velocity & $6.5^{\circ}$ \\
Target's yaw angle & $10^{\circ}$ & Beam width & $0^{\circ}$ \\
Polarization type & Torizontal polarization & Target's roll angle & $5^{\circ}$ \\
\hline
\end{tabular}

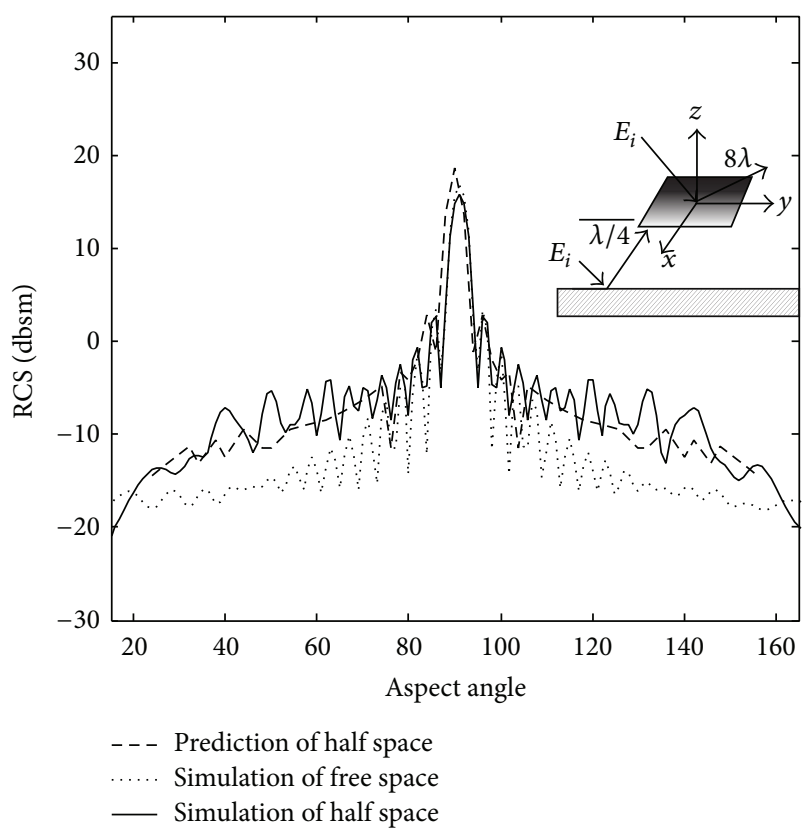

FIGURE 1: Comparison of prediction and simulation of software for an $8 \lambda \times 8 \lambda$ plane in half space (frequency $=10 \mathrm{GHz}$ ).

the demonstrated half-space GRECO is appropriate to the analysis of the scattering from the low-flying target.

Figure 1 shows a perfectly conducting flat plane $(8 \lambda \times$ $8 \lambda$ ) which is placed $\lambda / 4$ above the soil. $\lambda$ represents the wavelength in the vacuum. The relative dielectric permittivity of the soil is $\varepsilon_{r}=4.0$, and the relative magnetic permeability is $\mu_{r}=1.0$.

Figure 1 shows that the half space RCS and the free space RCS matches very well at the backscatter angle. However, at other incident angles, there are noticeable differences between them for 4-5 dbsm. Hence, the conclusion is drawn that it is necessary to consider the effect of the half-space environment.

\subsection{Multipath Effects}

2.2.1. Multipath Model. In detecting low altitude targets, the presence of reflections from sea or ground surface can cause multipath propagation. In some literatures, the implicit assumption is that only one reflection path exists [12]. However, in practice, four different paths, including direct-direct, direct-reflected, reflected-direct, and reflected-reflected, are contributing to the radar received signals, as shown analytically in Figure 2. The total echo from a low-flying target is thus a superposition of four ways of signals, each with a different path. Multipath propagation can cause signal cancellation in adaptive beamforming [23] and errors in lowangle radar tracking [24]. A highly deterministic multipath model is constituted in [25].

The complex RCS can be written as $\sqrt{\sigma} e^{j \phi_{t}}$. Based on the radar equation [1], the complex voltage coefficient of directdirect echo signal received by the radar is expressed as

$$
V_{1}(t)=\left(\frac{P_{t} \lambda^{2} \sigma}{(4 \pi)^{3} L}\right)^{1 / 2} \cdot \frac{f\left(\theta_{d}\right)}{R^{2}} e^{j\left(\phi_{r}+\phi_{t}\right)},
$$

where $P_{t}$ is the radar transmit peak power, $\lambda$ is radar wavelength, $\sigma$ is the RCS of target, $f\left(\theta_{d}\right)$ is radar antenna gain, $\theta_{d}$ is the angle between the target and radar beam boresight, $L$ is composite loss, $R$ is the distance from the radar to the center of target and can be obtained through the coordinates of radar and target, and $\phi_{r}$ is the total phase brought by the receiver system.

Since the path differences of the direct and multipath returns are usually less than the range resolution of the radar, the different returns are delivered into relevant range gates, resulting in fluctuations. Thus, the magnitude of the superposition of four signals can be expressed as

$$
\begin{aligned}
V_{\text {total }}= & \left|V_{1}+V_{2}+V_{3}+V_{4}\right|=\left(\frac{P_{t} \lambda^{2}}{(4 \pi)^{3} L}\right)^{1 / 2} \\
& \cdot \mid \frac{f\left(\theta_{d}\right)\left(\sigma_{1}\right)^{1 / 2}}{R^{2}} \\
& +\rho \frac{\left(f\left(\theta_{t}\right) \cdot f\left(\theta_{d}\right)\right)^{1 / 2}\left(\sigma_{2}\right)^{1 / 2}}{\left(R_{1}+R_{2}\right) R} e^{j\left(2 \pi \Delta L_{1} / \lambda\right)} \\
& +\rho \frac{\left(f\left(\theta_{t}\right) \cdot f\left(\theta_{d}\right)\right)^{1 / 2}\left(\sigma_{3}\right)^{1 / 2}}{\left(R_{1}+R_{2}\right) R} e^{j\left(2 \pi \Delta L_{2} / \lambda\right)} \\
& +\rho^{2} \frac{f\left(\theta_{t}\right)\left(\sigma_{4}\right)^{1 / 2}}{\left(R_{1}+R_{2}\right)^{2}} e^{j\left(2 \pi \Delta L_{3} / \lambda\right)} \mid .
\end{aligned}
$$




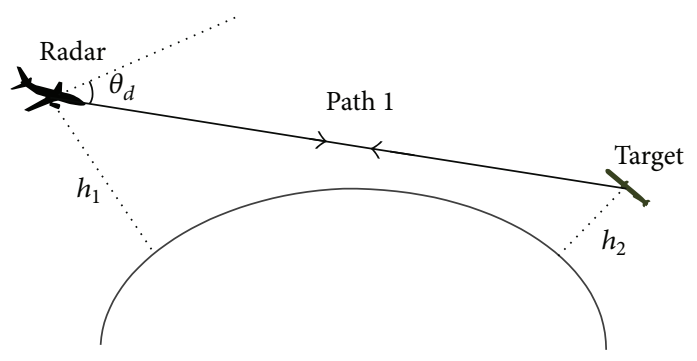

(a)

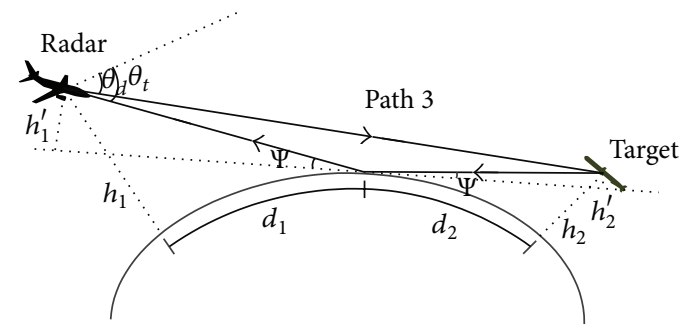

(c)

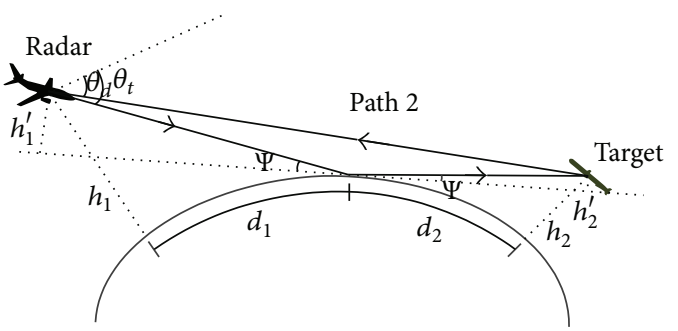

(b)

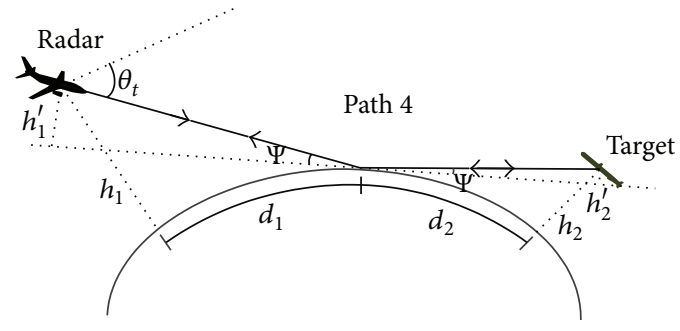

(d)

Figure 2: The radar-target geometry in the presence of multipath. (a) Path 1. (b) Path 2. (c) Path 3. (d) Path 4.

$\sigma_{1}, \sigma_{2}, \sigma_{3}$, and $\sigma_{4}$ are the magnitudes of complex RCS of different ways. $\rho$ is the reflection coefficient. $\Delta L_{1}, \Delta L_{2}$, and $\Delta L_{3}$ are the path length differences of three reflected rays compared to the direct. $R_{1}$ and $R_{2}$ are the distances of radar-to-reflection point and target-to-reflection point, respectively.

Multipath interference from a rough surface generally involves two components, which are specular and diffuse reflections. The specular reflection coefficient can be expressed as

$$
\rho_{s}=\rho_{0} R_{s} D,
$$

where $\rho_{0}$ is Fresnel reflection coefficient, $R_{s}$ is the specular scattering factor, and $D$ is divergence factor. For horizontal polarization, $\rho_{0}$ is expressed as [25]

$$
\rho_{0 H}=\frac{\sin \psi-\left(\varepsilon_{c}-\cos ^{2} \theta\right)^{1 / 2}}{\sin \psi+\left(\varepsilon_{c}-\cos ^{2} \theta\right)^{1 / 2}} .
$$

For vertical polarization, it is expressed as

$$
\rho_{0 V}=\frac{\varepsilon_{c} \sin \psi-\left(\varepsilon_{c}-\cos ^{2} \theta\right)^{1 / 2}}{\varepsilon_{c} \sin \psi+\left(\varepsilon_{c}-\cos ^{2} \theta\right)^{1 / 2}} .
$$

The divergence factor $D$ is taken into account due to the curvature of the Earth and given as

$$
D=\left[1+\frac{2 R_{1} R_{2}}{a\left(R_{1}+R_{2}\right) \sin \psi}\right]^{1 / 2} .
$$

The root mean square (RMS) value of the specular scattering factor is given as

$$
R_{s}=\exp \left[-2\left(\frac{2 \pi \sigma_{h} \sin \psi}{\lambda}\right)^{2}\right] .
$$

Here, $\sigma_{h}$ is the RMS value of the wave height.
The diffuse reflection coefficient is given as

$$
\rho_{D}=\rho_{0} R_{D},
$$

where $\rho_{0}$ is the Fresnel reflection coefficient defined in (9) and (10) and $R_{D}$ is the diffuse scattering coefficient [26].

The Rayleigh roughness criterion from optical theory is commonly used to estimate the maximum surface irregularity that will not significantly lower the reflection coefficient $[27,28]$ :

$$
\Delta h<\frac{\lambda}{8 \sin \psi},
$$

where $\Delta h$ is the maximum peak-to-trough variations and $\psi$ is the grazing angle of reflection point. If (14) is satisfied, the surface can behave as an essentially smooth dielectric, where the specular reflection will occur.

2.2.2. Estimation Approaches Based on Monte Carlo Methods. The one-way propagation experimental data for a variety of terrain types are presented in [13], which indicate a statistical simulation is required for the signal strength in practice. References $[1,2,11]$ treat the path length differences $\Delta L_{1}, \Delta L_{2}$, and $\Delta L_{3}$ as deterministic variables. However, the errors caused by multipath geometry calculation are so large with respect to the wavelength that they can confuse the interference. For a radar operating at $1 \mathrm{GHz}$, an error of 0.15 meter will cause a phase error of $180^{\circ}$. Also, due to airborne radar working at a large height, the gracing angle of the target is usually so large [1] that it causes a significant difference of direction between the direct and reflected ray, which makes the phases of scattering fields of the direct and reflected ray quite different from each other. Hence, the phase factors will be treated as variables which satisfy some PDF. The statistic peculiarity of echo is calculated with Monte Carlo methods. 
Monte Carlo methods are algorithm for solving various kinds of computational problems by using random numbers [29]. It is a means of treating mathematical problems by finding a probabilistic analog and then obtaining approximate answers to this analog by some experimental sampling procedure. The solution of a problem by this method is closer in spirit to physical experiments than to classical numerical techniques. In our calculations, the number of samples is increased to achieve a fairly regular distribution. As for the error from the Monte Carlo sampling technique, it can be made negligible by making the number of samples sufficiently large.

To simulate the instantaneous amplitude from echo signals with Monte Carlo method, firstly the probabilistic model according to the problem should be established. From formula (7), the phase factors can be rewritten as

$$
\begin{aligned}
& \varphi_{1}=\left(\frac{2 \pi \Delta L_{1}}{\lambda}\right) \bmod (2 \pi), \\
& \varphi_{2}=\left(\frac{2 \pi \Delta L_{2}}{\lambda}\right) \bmod (2 \pi), \\
& \varphi_{3}=\left(\frac{2 \pi \Delta L_{3}}{\lambda}\right) \bmod (2 \pi),
\end{aligned}
$$

where $\varphi_{1}, \varphi_{2}$, and $\varphi_{3}$ are the remainder of phase differences divide $2 \pi$ of three reflected rays compared to the direct. For airborne radar, we know that the path length differences of three reflected rays compared to the direct can far exceed the wave length, so $\varphi_{1}, \varphi_{2}$, and $\varphi_{3}$ can be supposed to three sequences of random numbers from a uniform distribution in $(0,2 \pi)$.

And expression (7) can be derived as

$$
\begin{aligned}
V_{\text {total }}= & \left|V_{1}+V_{2}+V_{3}+V_{4}\right|=\left(\frac{P_{t} \lambda^{2} \sigma}{(4 \pi)^{3} L}\right)^{1 / 2} \\
& \cdot \mid \frac{f\left(\theta_{d}\right)\left(\sigma_{1}\right)^{1 / 2}}{R^{2}}+\rho \frac{\left(f\left(\theta_{t}\right) \cdot f\left(\theta_{d}\right)\right)^{1 / 2}\left(\sigma_{2}\right)^{1 / 2}}{\left(R_{1}+R_{2}\right) R} e^{j \varphi_{1}} \\
& +\rho \frac{\left(f\left(\theta_{t}\right) \cdot f\left(\theta_{d}\right)\right)^{1 / 2}\left(\sigma_{3}\right)^{1 / 2}}{\left(R_{1}+R_{2}\right) R} e^{j \varphi_{2}} \\
& +\rho^{2} \frac{f\left(\theta_{t}\right)\left(\sigma_{4}\right)^{1 / 2}}{\left(R_{1}+R_{2}\right)^{2}} e^{j \varphi_{3}} \mid .
\end{aligned}
$$

With the most widely used mixed congruence method [30], we generate three sequences of random numbers $a_{i}, b_{i}$, and $c_{i}(i=1,2, \ldots, N)$, which is uniformly distributed in the interval $(0,1)$,

$$
\begin{gathered}
a_{i}=\frac{\left[\left(m_{a} a_{i-1}+n_{a}\right) \bmod M\right]}{M}, \\
b_{i}=\frac{\left[\left(m_{b} b_{i-1}+n_{b}\right) \bmod M\right]}{M}, \\
c_{i}=\frac{\left[\left(m_{c} c_{i-1}+n_{c}\right) \bmod M\right]}{M}, \\
i=1,2, \ldots, N,
\end{gathered}
$$

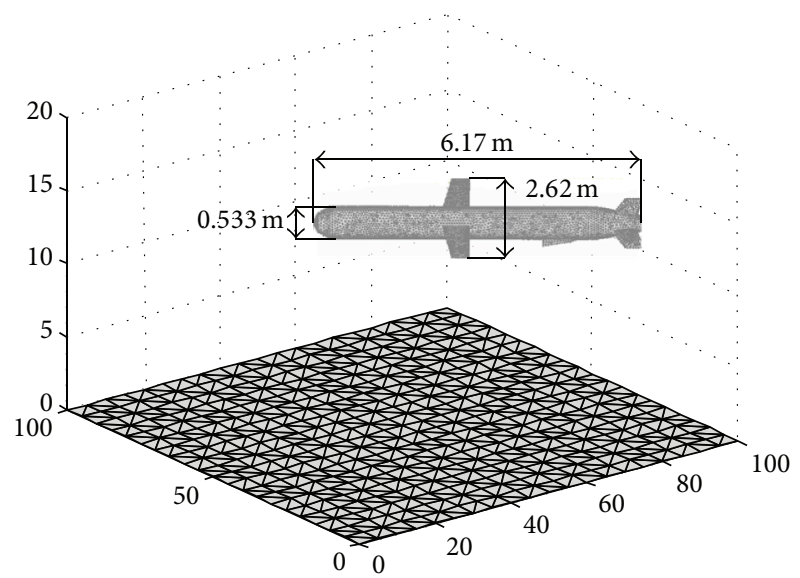

FIgURE 3: The missile is placed $20 \mathrm{~m}$ above the sea.

where $m_{a}, m_{b}$, and $m_{c}$ are the multipliers; $n_{a}, n_{b}$, and $n_{c}$ are the increment; $M$ is the modulus.

Given $\varphi_{1}=2 \pi a_{i}, \varphi_{2}=2 \pi b_{i}$, and $\varphi_{3}=2 \pi c_{i}$ in formula (16), run the Monte Carlo simulation $\mathrm{n}$ times to estimate the echo signals from low altitude targets accurately based on actual environment for airborne radar.

By using a sufficient number of samples, a probability distribution is obtained to describe echo signals. The statistical characteristics of the echo signals can be illustrated at last.

\section{Results and Discussion}

The missile is adopted in the simulation as shown in Figure 3 with its RCS in Figure 4.

The PDF of magnitude of echoes is shown in Figure 5 when the target is flying at a height of $20 \mathrm{~m}$ above the sea. According to the radar equation, the magnitude of direct ray is $6.4416 \times 10^{-7} \mathrm{~V}$. Figure 5 indicates that the magnitude of echoes can be different from direct ray due to multipath.

The magnitudes of direct echoes in the situations of different target's heights are shown in Figure 6, and the PDFs of magnitude of echoes normalized to the direct ray in the same situations are shown in Figure 7. As can be seen in Figure 6, the magnitudes of direct echoes vary with target's heights. The prime reason for that is the target RCS's variation caused by the different azimuth angle $\varphi$ and the elevation angle $\theta$ with respect to the coordinate system of the target. Figure 7 shows the influences of multipath at different heights. In the conditions of the target flying at low heights (about a few hundred meters), the PDFs vary little with the heights, while the normalized magnitude of echoes with respect to the peak of the PDF reduces a little. As illustrated in Figure 7, when the target is at heights of below $2000 \mathrm{~m}$, the magnitudes of echoes normalized to the direct ray vary mainly between 0 and 4 . The peak probability densities are in the range of $0-1$ and the corresponding normalized magnitudes of echoes are in the range of 1-2. These results in Figure 7 are similar to the ones in Figures 14 and 15 of [13], which make the demonstrated algorithm valid in this paper. However, the terrain in this paper was produced from the digital feature analysis data 


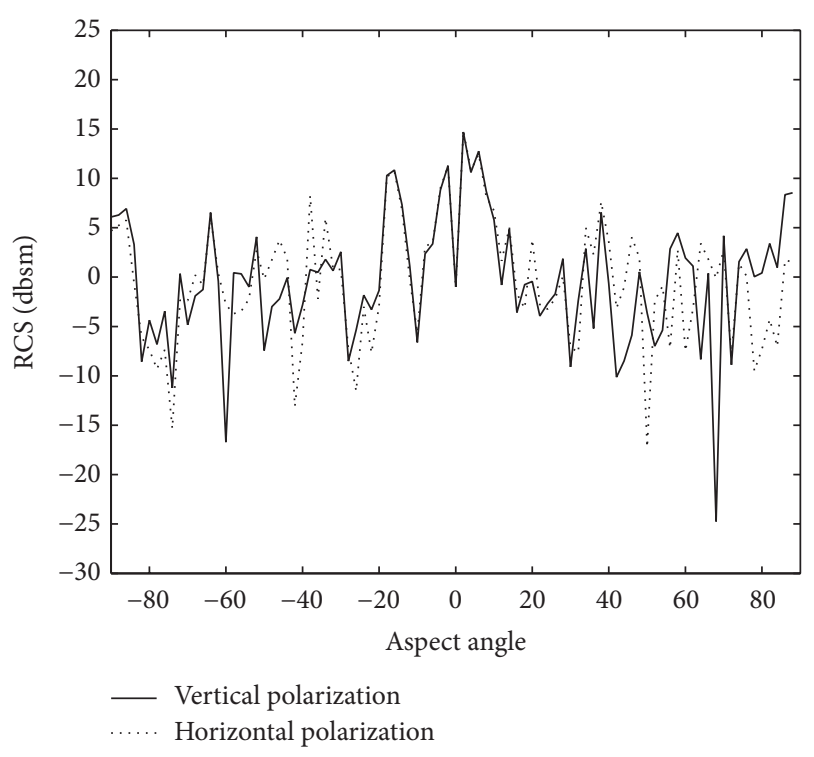

FIgURE 4: The missile's RCS with frequency of $0.4 \mathrm{GHz}$.

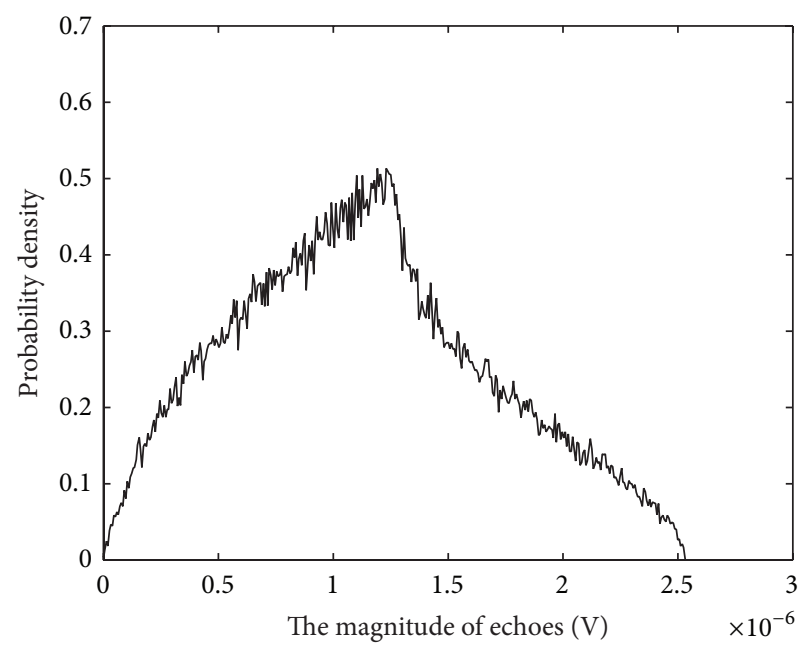

Figure 5: The PDF, versus magnitude of echoes, for the target at a height of $20 \mathrm{~m}$ above the sea.

(DFAD), yet, in [13], the desert terrain for simulations was produced from the defense mapping agency (DMA) data; the experiments were made in New Mexico and Northern Maine. The differences between the results in this paper and those in [13] are mainly caused by the differences in the terrains, the frequencies, the geometries, and attitudes of the targets, the heights of the airborne radars and the targets, the number of samples, and so forth. As the target is flying higher, the influences of multipath become less to the echoes until the indirect ways disappear. In practice, if the target height is at least moderately large, the range resolution of the radar is less than the path differences of the direct and multipath returns, so the radar is able to resolve them. As radar height decreases to a height of ground based radar, the difference in angle between reflected and direct paths can be ignored,

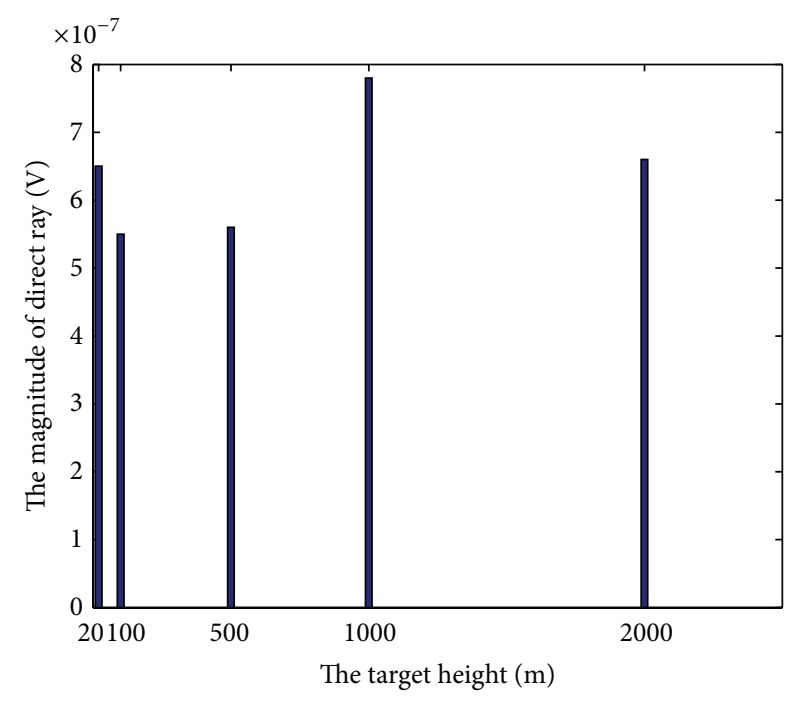

FIGURE 6: The magnitude of direct ray versus the target height.

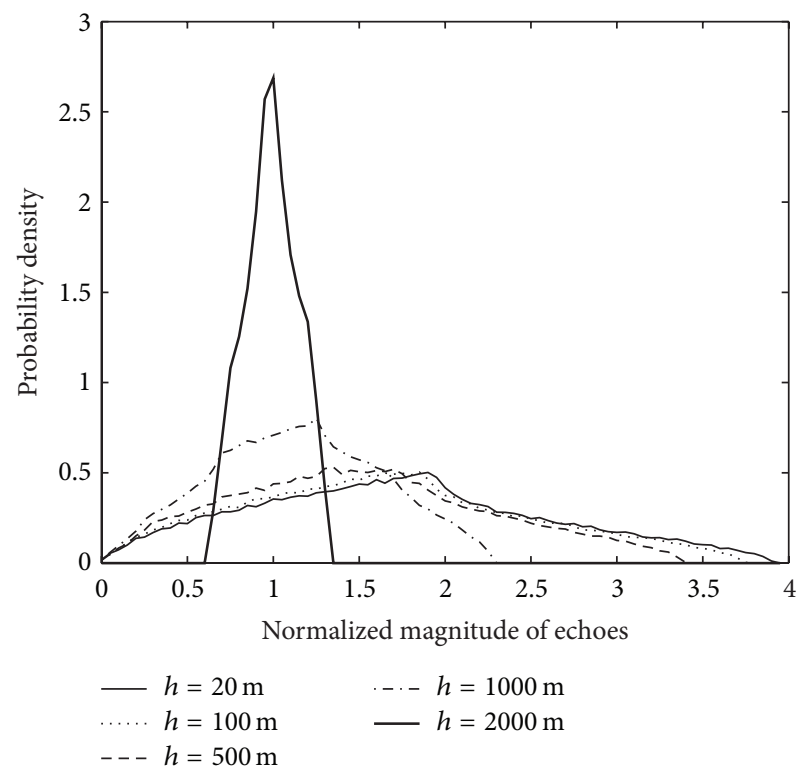

FIGURE 7: The PDF of magnitude of echoes at different target's heights.

which causes the fluctuations in phase disappearing for lowflying targets, and the PDF of magnitude of echoes is similar to Figure 6 of [12].

\section{Conclusions}

For airborne radar to detect low altitude targets, a demonstrated hybrid method based on probability, statistics, and computational electromagnetics is proposed in this paper to give a statistical simulation of echo signals based on actual environment. The half-space physical optics combined with the graphical-electromagnetic computing method is employed to calculate the RCS of low-flying targets sufficiently accurately and efficiently in half space. Considering 
the fluctuations due to the multipath effects, the phase factors are modified with the Monte Carlo method. With this method, the target echo signals from low altitude targets can be obtained accurately for the radar simulation system, and the statistic characteristics of echo signals can be accurately and properly simulated. The demonstrated method in this paper is of great help for the radar researcher to evaluate the detection probability and false alarm probability of special targets, the signal-to-noise ratio (SNR) of emission pulses, and so on.

\section{Conflict of Interests}

The authors declare that there is no conflict of interests regarding the publication of this paper.

\section{References}

[1] M. Long, Airborne Early Warning System Concepts, Artech, Boston, Mass, USA, 1992.

[2] W. Morchin, Airborne Early Warning Radar, Artech House, Norwell, Mass, USA, 1990.

[3] L.-X. Guo, A.-Q. Wang, and J. Ma, "Study on EM scattering from 2-D target above 1-D large scale rough surface with low grazing incidence by parallel MOM based on PC Clusters," Progress in Electromagnetics Research, vol. 89, pp. 149-166, 2009.

[4] M. I. Skolnik, Introduction to Radar Systems, McGraw-Hill, New York, NY, USA, 3rd edition, 2001.

[5] Y. Qu, G. S. Liao, S. Q. Zhu, and X. Y. Liu, "Pattern synthesis of planar antenna array via convex optimization for airborne forward looking radar," Progress in Electromagnetics Research, vol. 84, pp. 1-10, 2008.

[6] S. H. Lim, J. H. Han, S. Y. Kim, and N. H. Myung, "Azimuth beam pattern synthesis for air-borne sar system optimization," Progress in Electromagnetics Research, vol. 106, pp. 295-309, 2010.

[7] Y.-L. Chang, C.-Y. Chiang, and K.-S. Chen, "SAR image simulation with application to target recognition," Progress in Electromagnetics Research, vol. 119, pp. 35-57, 2011.

[8] M. Zhang, Y. W. Zhao, H. Chen, and W. Q. Jiang, "Sar imaging simulation for composite model of ship on dynamic ocean scene," Progress in Electromagnetics Research, vol. 113, pp. 395412, 2011.

[9] S. Qiao, Z. G. Shi, K. S. Chen et al., "A new architecture of UWB radar utilizing microwave chaotic signals and chaos synchronization," Progress in Electromagnetics Research, vol. 75, pp. 225-237, 2007.

[10] M.-J. Wang, Z.-S. Wu, Y.-L. Li, and G. Zhang, "High resolution range profile identifying simulation of laser radar based on pulse beam scattering characteristics of targets," Progress in Electromagnetics Research, vol. 96, pp. 193-204, 2009.

[11] J. G. Teti Jr., "Wide-band airborne radar operating considerations for low-altitude surveillance in the presence of specular multipath," IEEE Transactions on Antennas and Propagation, vol. 48, no. 2, pp. 176-191, 2000.

[12] S. L. Wilson and B. D. Carison, "Radar detection in multipath," IEE Proceedings Radar, Sonar and Navigation, vol. 146, no. 1, pp. 45-52, 1999.

[13] L. M. Zurk, "Experimental observation and statistics of multipath from terrain with application to overland height finding,"
IEEE Transactions on Antennas and Propagation, vol. 47, no. 1, pp. 121-131, 1999.

[14] Y. B. Tao, H. Lin, and H. J. Bao, "From CPU to GPU: GPUbased electromagnetic computing," Progress in Electromagnetics Research, vol. 81, pp. 1-19, 2008.

[15] B. Etkin and L. D. Reid, Dynamics of Flight: Stability and Control, John Wiley \& Sons, New York, NY, USA, 1996.

[16] X. F. Li, Y. J. Xie, and R. Yang, "Bistatic RCS prediction for complex targets using modified current marching technique," Progress in Electromagnetics Research, vol. 93, pp. 13-28, 2009.

[17] X. F. Li, Y. J. Xie, P. Wang, and T. M. Yang, "High-frequency method for scattering from electrically large conductive targets in half-space," IEEE Antennas and Wireless Propagation Letters, vol. 6, pp. 259-262, 2007.

[18] K. A. Michalski and D. Zheng, "Electromagnetic scattering and radiation by surfaces of arbitrary shape in layered media. I. Theory," IEEE Transactions on Antennas and Propagation, vol. 38, no. 3, pp. 335-344, 1990.

[19] H. Bağci, A. E. Yilmaz, V. Lomakin, and E. Michielssen, "Fast solution of mixed-potential time-domain integral equations for half-space environments," IEEE Transactions on Geoscience and Remote Sensing, vol. 43, no. 2, pp. 269-279, 2005.

[20] R. C. Acar and G. Dural, "Complete set of closed-form Green's functions for cylindrically layered media," in Proceedings of the IEEE Antennas and Propagation Society International Symposium 2006, pp. 2863-2866, 2006.

[21] J. M. Rius, M. Ferrando, and L. Jofre, "High-frequency RCS of complex radar targets in real-time," IEEE Transactions on Antennas and Propagation, vol. 41, no. 9, pp. 1308-1319, 1993.

[22] E. F. Knott, J. F. Shaeffer, and M. T. Tuley, Radar Cross Section, SciTech Publishing, Raleigh, NC, USA, 2004.

[23] B. Widrow, K. M. Duvall, R. P. Gooch, and W. C. Newman, "Signal cancellation phenomena in adaptive antennas: causes and cures," IEEE Transactions on Antennas and Propagation, vol. 30, no. 3, pp. 469-478, 1982.

[24] W. D. White, "Low angle radar tracking in the presence of multipath," IEEE Transactions on Aerospace and Electronic Systems, vol. 10, no. 6, pp. 335-352, 1974.

[25] T. Lo and J. Litva, "Use of a highly deterministic multipath signal model in low-angle tracking," IEE Proceedings, Part F: Radar and Signal Processing, vol. 138, no. 2, pp. 163-171, 1991.

[26] P. Beckmann and A. Spizzichino, The Scattering of ElectroMagnetic Waves from Rough Surfaces, Artech House, Norwood, Mass, USA, 1987.

[27] C. I. Beard, "Coherent and incoherent scattering of microwaves from the ocean," IEEE Transactions on Antenna and Propagation, vol. 9, no. 5, pp. 470-483, 1961.

[28] N. Pinel, C. Bourlier, and J. Saillard, "Degree of roughness of rough layers: extensions of the Rayleigh roughness," Progress in Electromagnetics Research B, no. 19, pp. 41-63, 2010.

[29] M. Mishra and N. Gupta, "Monte Carlo integration technique for the analysis of electromagnetic scattering from conducting surfaces," Progress in Electromagnetics Research, vol. 79, pp. 91106, 2008.

[30] M. Á. Herrador, A. G. Asuero, and A. G. González, "Estimation of the uncertainty of indirect measurements from the propagation of distributions by using the Monte-Carlo method: an overview," Chemometrics and Intelligent Laboratory Systems, vol. 79, no. 1-2, pp. 115-122, 2005. 

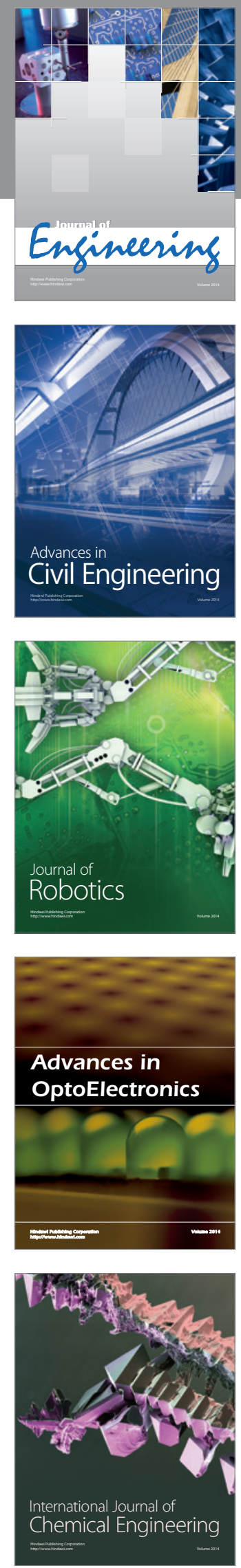

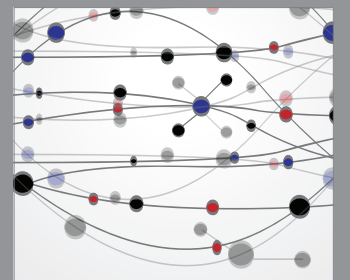

The Scientific World Journal
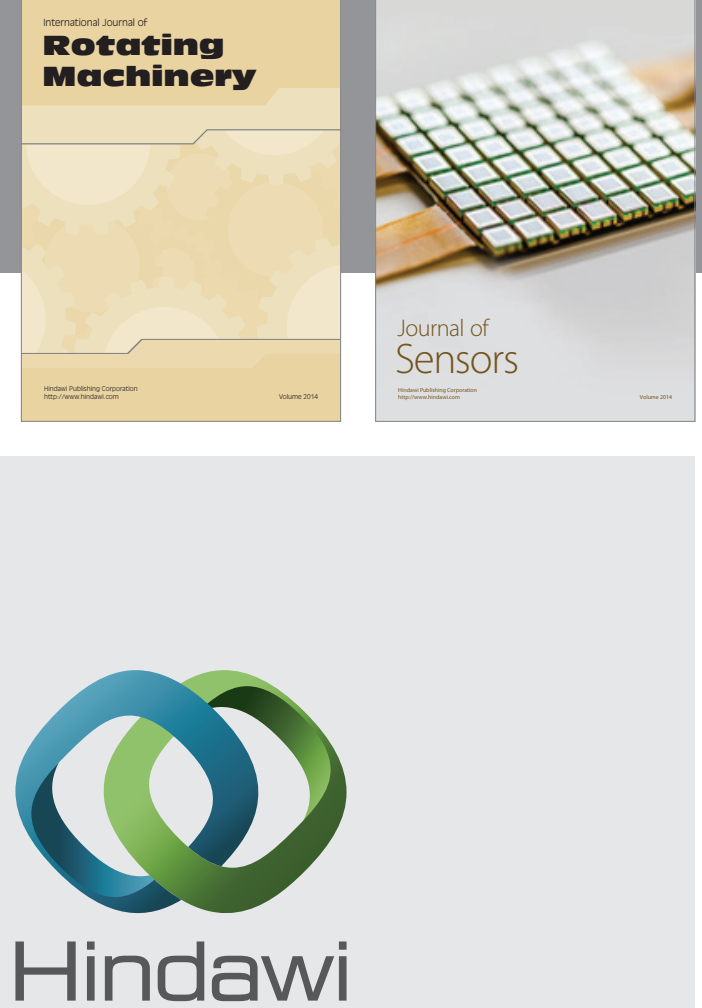

Submit your manuscripts at http://www.hindawi.com
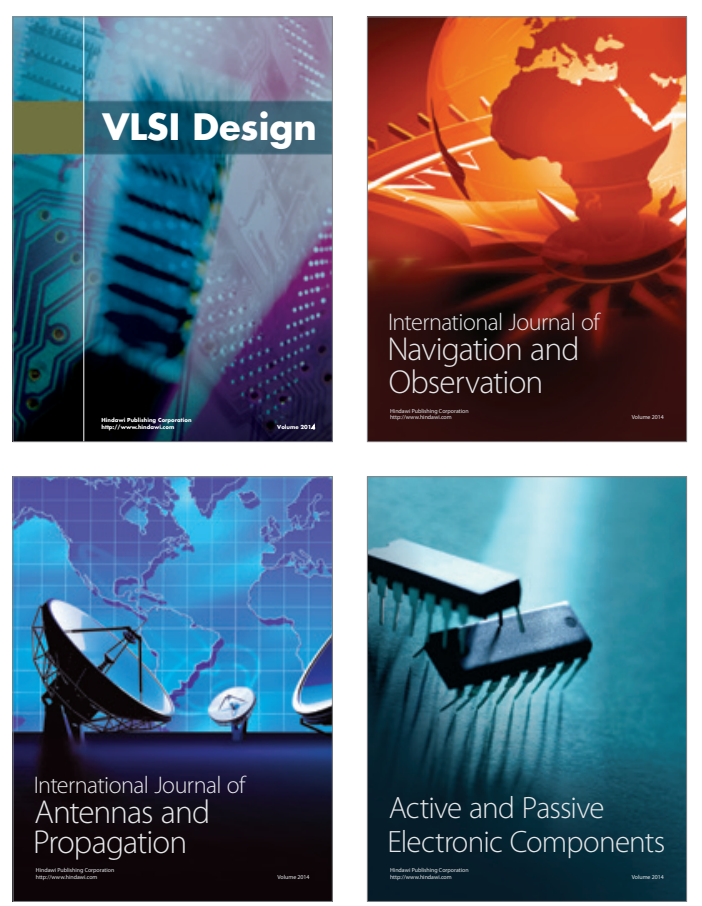
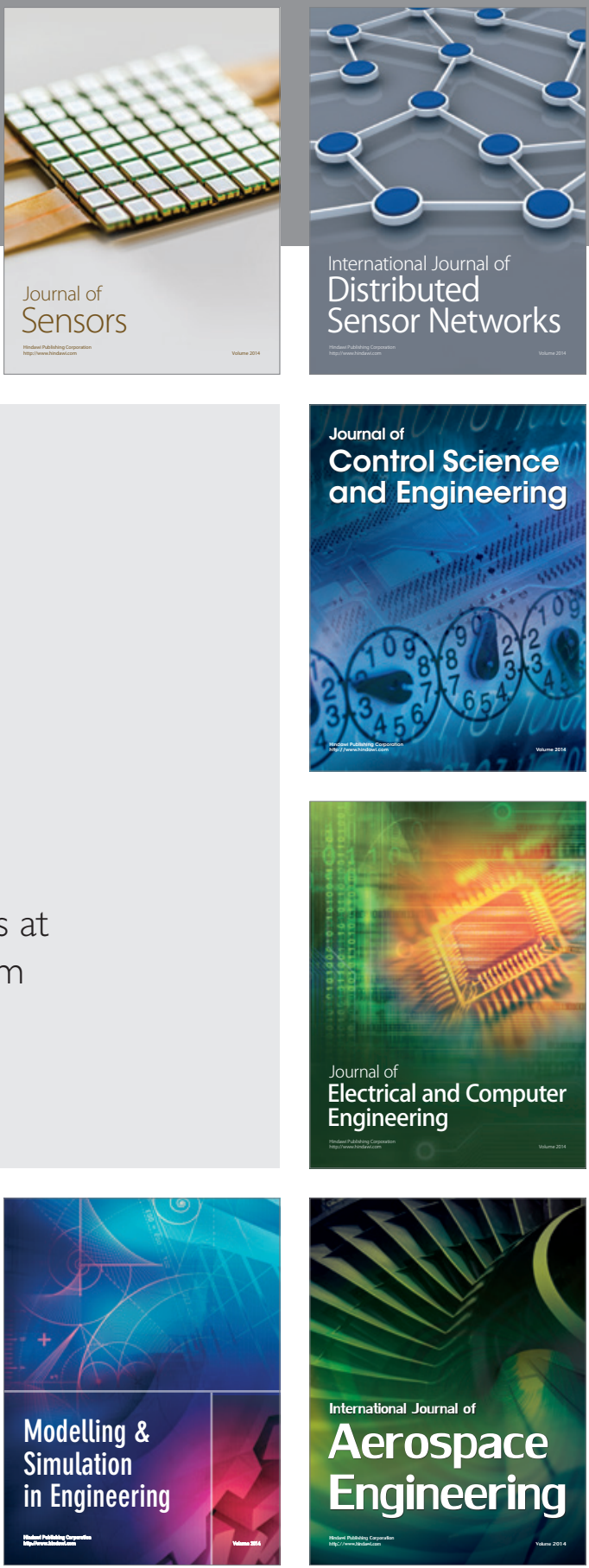

Journal of

Control Science

and Engineering
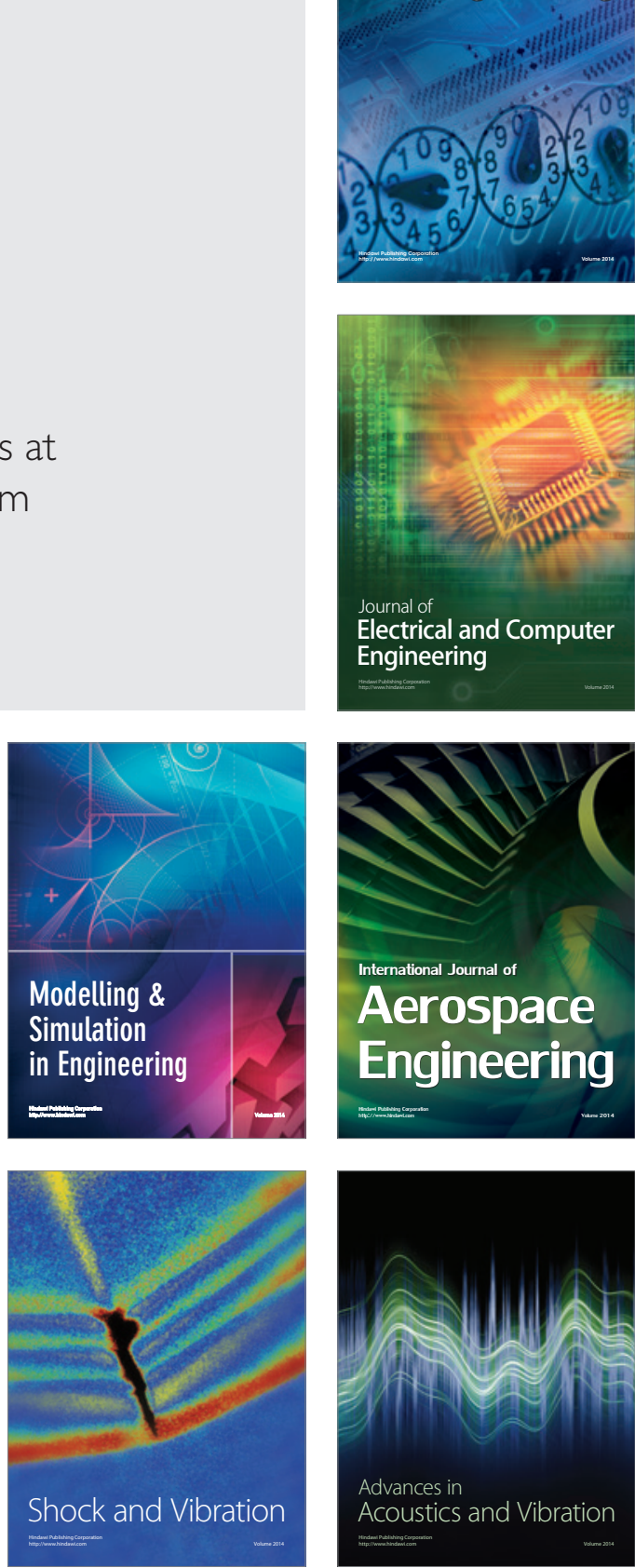